\title{
Button-hole: técnica de punción de elección en hemodiálisis domiciliaria
}

\author{
Laura Baena Ruíz \\ Unidad de nefrología y diálisis. Hospital Universitario del Henares. Madrid
}

\section{Introducción}

Las Fístulas arterio-venosas (FAV) son el acceso vascular de elección en los pacientes en tratamiento con hemodiálisis. Para la enfermería nefrológica es imprescindible un conocimiento en profundidad de las mismas, así como de las diferentes técnicas de punción existentes.

Las técnicas de punción habituales son de sobra conocidas por todo el personal de enfermería dedicado a los pacientes renales, pero existe una técnica poco extendida que puede sernos de gran utilidad: el ButtonHole.

Esta técnica, a pesar de llevar tiempo practicándose, en España es poco conocida,

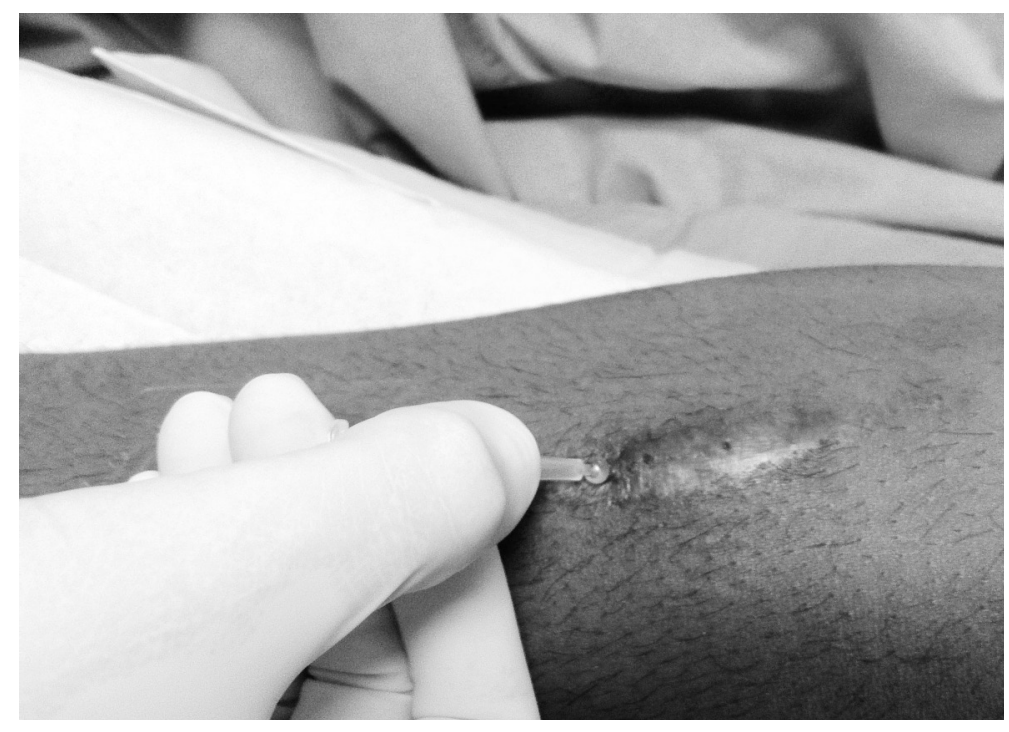
pero puede resultar beneficiosa para muchos pacientes en los que nos encontramos con problemas de punción, en FAV profundas o con poco trayecto o, como en nuestro caso, en pacientes candidatos a realizarse el tratamiento de hemodiálisis domiciliaria (HDD).

\section{Objetivos}

El objetivo principal de la aplicación de esta técnica en nuestro centro fue el de facilitar la punción a nuestro primer paciente candidato a HDD. Como objetivo secundario nos planteamos extender la técnica a pacien-

\begin{tabular}{|c|}
\hline Correspondencia: \\
Laura Baena Ruíz \\
Unidad de nefrología. Hospital del Henares \\
Avenida Marie Curie S/N, 28820 \\
Coslada, Madrid \\
E-mail: Iaura.baena@salud.madrid.org \\
\hline
\end{tabular}

Imagen 1

tes que reciben tratamiento hospitalario, bien porque fueran candidatos a realizarse autopunción o por presentar dificultades en la punción de manera reiterada.

\section{Caso clínico}

En nuestro centro se planteó la posibilidad de aplicar la técnica del Button-Hole o técnica del ojal al iniciar la técnica de HDD para facilitar al paciente la realización de la punción en el domicilio minimizando complicaciones.

La técnica consiste en la creación de un túnel subcutáneo como acceso permanente e invariable a la FAV, a través del cual se puncionará con aguja roma una vez formado. La primera punción ha de realizarse con aguja convencional, eligiendo la zona teniendo en cuenta que la separación entre las dos punciones debe ser de al menos $6 \mathrm{~cm}$ y que no se realice en zona aneurismática. Tras realizar la sesión de hemodiálisis se extraen ambas agujas coagulando sin ningún tipo de apósito hemostático. 


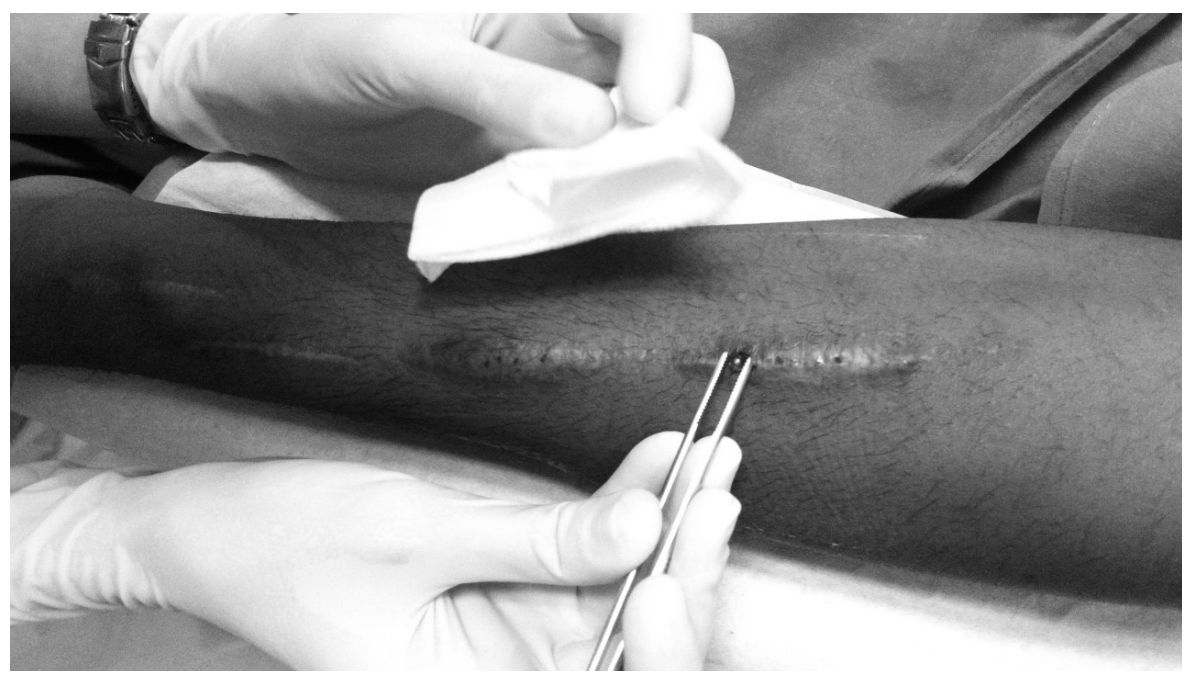

Imagen 2

Pasados casi años de su primera hemodiálisis en domicilio no ha presentado ningún problema de punción ni relacionado con la FAV, por lo que hemos extendido la técnica a pacientes que realizan hemodiálisis hospitalaria en sala de crónicos. El planteamiento de la unidad es extender la técnica a todos los pacientes que se puedan beneficiar de ella, ya que en todos los pacientes en la que se está practicando se ha reducido notoriamente el

Una vez que el paciente ha coagulado se desinfecta la zona con clorexhidina y se deja actuar antes de aplicar el Kit para la estabilización del túnel. El Kit de estabilización se colocará con técnica estéril (Imagen 1). Se dejará implantado hasta la siguiente sesión, cubriéndose con apósito trasparente. En la siguiente sesión se retira el apósito transparente sin retirar el Kit, se desinfecta con clorhexidina y después se retira el Kit de con técnica estéril con ayuda de unas pinzas (Imagen 2). El túnel quedará visible (Imagen 3 ). Se canalizará el acceso vascular con aguja roma. Debemos aplicar el Kit de estabilización durante 506 sesiones, siempre con la misma técnica y por la misma enfermera, previamente entrenada.

Una vez que el túnel está estabilizado se realizará la punción con aguja roma, retirando previamente la costra que se forma en el orificio tras la coagulación, con técnica limpia, desinfectando la zona antes y después de la retirada de la costra (doble asepsia). La coagulación será siempre manual y sin aplicación de ningún tipo de hemostático para minimizar los riesgos de infección y la posibilidad de que el túnel quede taponado por el hemostático.

En el caso de nuestro paciente en hemodiálisis domiciliaria se necesitaron 5 días, con hemodiálisis diaria, para la formación completa de los dos túneles subcutáneos. Tras otros cinco días de punción (siempre por la misma enfermera), comenzó a realizarse el entrenamiento de la autopunción, y en 5 días más el paciente se autopuncionaba sin incidencias. Una semana después el paciente comenzó a realizarse la hemodiálisis en su domicilio. dolor a la punción, se han reducido las cicatrices visibles de punciones repetidas y no ha habido ningún episodio de punción fallida en ninguno de ellos.

En el caso de nuestro paciente en HDD no ha presentado ninguna infección, ningún episodio de extravasación y ninguna punción fallida. Los pequeños aneurismas que presentaba previamente no han aumentado de tamaño, y los flujos de sangre que se logran son los mismos. Las cifras de presión arterial y venosa se han mantenido en el mismo rango que con la punción escalonada.

El paciente refiere que la técnica del Button-hole le ha facilitado en gran medida la autopunción, ya que ha eliminado el dolor y le da mucha seguridad el saber que no es posible fallar la punción ni producirse ningún daño a su acceso vascular.

Imagen 3

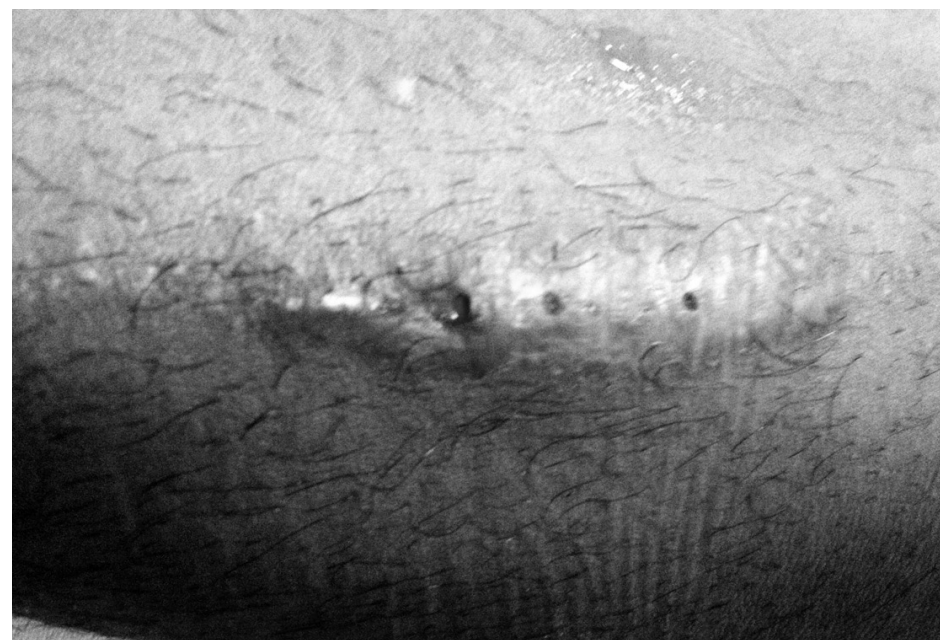




\section{Conclusiones}

La técnica del Button-Hole o Técnica del 0jal es poco conocida en el ámbito de la enfermería nefrológica española, pero en otros lugares de Europa y Asia se realiza de manera muy habitual con buenos resultados.

Una de las tareas fundamentales de la enfermera nefrológica es el de preservar los accesos vasculares de nuestros pacientes, ya que de ellos dependen sus vidas. Esta técnica de punción es la técnica de elección, sin duda, para la hemodiálisis domiciliaria, pero no es exclusiva para esta modalidad, ya que la mayoría de los pacientes con FAV se podrían beneficiar de ella independientemente de donde realicen las sesiones de hemodiálisis.

Para poder aplicar esta técnica de punción es necesaria la formación del personal de enfermería, requiriendo implicación, ya que la enfermera que realiza el túnel debe coordinarse con el paciente para que sea el mismo profesional el que realice el túnel y forme al paciente en la autopunción.

\section{Bibliografía}

1. Figueiredo $A E$, Viegas $A$, Monteiro M, Poli-de-Figueiredo $C E$. Research into pain perception with arteriovenous fistula (avf) cannulation. J Ren Care. 2008 Dec;34(4):169-72.

2. Kim MK, Kim HS. Clinical effects $f$ buttonhole cannulation method $\mathrm{n}$ hemodialysis patients. Hemodial $\mathrm{nt}$. 2012;32.
3. Ludlow V. Buttonhole cannulation in hemodialysis: improved outcomes and increased expense -is it worth it?. CANNT J. 2010; 20 (1): 29-37.

4. Murcutt, $G$, et al Buttonhole cannulation: should this become the default technique for dialysis patients with native fistulas? Summary of the EDTNA/ERCA Journal Club discussion Autumn 2007 Journal of Renal Care, 34 (2) p. 101-108.

5. Sukthinthai N, Sittipraneet $A$, Tummaittayangkoon $B$, Vasuvattakul S, Chanchairujira T. Buttonhole technique better tan area puncture technique on hemostasis and pain associated with needle cannulation. $\mathrm{J}$ Med Assoc Tahi. 2012; 2: 208-12.

6. Twardowski, Z, Kubara, H. Different sites versus constant site of needle insertion into arteriovenous fistulas for treatment by repeated dialysis. Dial \& Transpl. 1979; 8: 978-980.

7. Twardowski, Z. Utility of the buttonhole cannulation method for haemodialysis patients with arteriovenous fistulas. Nature Clinical Practice Nephrology, (2007) 3: 12 p. 648-649.

8. Van Loon M, Goovaerts $T$, Kessels $A$, Van der Sande F, Tordoir JHM. Buttonhole needling of haemodialysis arteriovenous fistulae results in less complications and interventions compared to the rope-ladder technique. Nephrol Dial Transplant (2010); 25: 225-230.

9. Verhallen AM, et al Cannulating in haemodialysis: rope ladder or buttonhole technique? Nephrology Dialysis \& Transplantation, (2007) 22: 2601-2604. 